\title{
Spare hypoxia, spoil the child?
}

\author{
Jason Boehme, MD, and Emin Maltepe, MD PhD \\ Departments of Pediatrics and Biomedical Sciences, UCSF, San Francisco, California, USA.
}

\begin{abstract}
Clinical vignette: An 8-year-old boy presents to the pediatric ICU after two days of cough with increasing secretions. The patient is progressing to respiratory failure and requires noninvasive mechanical ventilation. His past medical history is remarkable for premature birth at 25 and 6/7 weeks gestational age, cerebral palsy, developmental delay, epilepsy, and gastrostomy tube dependence. His chest $x$-ray is remarkable for multifocal opacities that are consistent with atelectasis. A complete blood count reveals a wbc count of 9.2 with a normal differential, $\mathrm{Hg}$ of 11.7, and platelet count of 276,000 . A respiratory viral panel from a nasal swab returns positive for rhinovirus. Additional patient history from the parents uncovers that he has been hospitalized three times over the course of the past 2 years with a similar presentation.
\end{abstract}

\section{Current knowledge}

The patient in this scenario illustrates a common and increasingly frequent clinical scenario in pediatric hospitals and wards. The most recent published admissions data from The Pediatric Health Information System database show that children with significant chronic medical conditions accounted for $19.2 \%$ of hospitalized patients, but a remarkable $48.9 \%$ of hospital days and $53.2 \%$ of hospital charges (1). Within this group, the most common primary chronic diagnosis was cerebral palsy.

The increasing survival of premature infants, particularly those at the extremes of prematurity and low birth weight, has resulted in a marked increase in the number of premature infants surviving into childhood. Unfortunately, many individuals born prematurely suffer chronic neurologic impairments (2). Recent data indicate that approximately $10 \%$ to $15 \%$ of extremely premature infants will go on to exhibit cerebral palsy, with an incidence that is inversely related to gestational age (3). Up to $50 \%$ will later exhibit cognitive or behavioral deficits (4). High-grade intraventricular hemorrhage (IVH) is a rare but important adverse event that affects this patient population; however, the most common radiologic and neuropathologic findings correlate these functional deficits to periventricular leukomalacia (PVL) and its accompanying neuronal/axonal abnormalities. PVL most broadly refers to a pattern of diffuse cerebral white-matter injury, with specific areas of necrosis and loss of cellular elements within the deep periventricular white matter (5). In the classic PVL description, these necrotic areas were initially quite large and subsequently evolved over time into macrocystic lesions (Figure 1). Thankfully, this type of PVL presentation has become more of a historical note, with recent imaging studies showing that severe PVL has a modern incidence of less than $5 \%(6,7)$. In modern practice, it is far more common for areas of necrosis to be microscopic and progress to foci of glial scarring (so-called microcystic PVL). Moreover, the diffuse white-matter abnormalities are characterized by astrogliosis, microgliosis, and altered numbers and maturation of cells of the oligodendrocyte lineage (8). Imaging studies indicate that $50 \%$ or more of very low birth weight

Related Article: p. 1319

Conflict of interest: The authors have declared that no conflict of interest exists.

Reference information: J Clin Invest. 2015;125(3):965-967. doi:10.1172/JCI80820.

(VLBW) infants present with manifestations that are consistent with PVL (5).

PVL pathogenesis is multifactorial and incompletely understood. Human, animal, and in vitro data suggest that upstream physiologic derangements converge to cause the death and/or maturational arrest of oligodendrocyte precursors (preOLs), ultimately leading to the characteristic abnormalities of white-matter myelination. PreOLs are vulnerable to reactive oxygen and nitrogen species, excitatory molecules, and inflammatory mediators at developmentally specific and temporally restricted periods, helping to account for the highest incidence of PVL within infants born within a specific window of early prematurity $(2,8)$. The upstream events most commonly implicated in triggering these downstream sequelae are hypoxia/ischemia and inflammation. Interestingly, the mechanism whereby ischemia leads to PVL remains elusive. It is generally attributed to peculiarities of the arterial architecture serving the periventricular white matter coupled with immature autoregulation of cerebral blood flow (8-10). While the anatomy of these specific distal arterial fields is fairly well described, the idea that this predisposes the periventricular deep white matter to vascular insufficiency is merely inferred (9-11). Other regions perfused by end arteries without rich vascular anastomoses are not as prone to ischemic injury. Additionally, this theory is complicated by the lack of an animal model that recapitulates the focal changes of clinical PVL. Exposure to global hypoxia and/or ischemia in rodent and large animal models certainly results in widespread white-matter injury with frank infarction (12). In other models, to achieve more selective involvement of white matter requires adherence to an extremely narrow window of hypoxic exposure before gray matter injury ensues (2).

\section{Research advances}

In this issue, Licht, Dor-Wollman, and colleagues report on their generation of a novel murine model that recapitulates specific temporal and regional phenotypes analogous to those of human PVL (13). Spe- 


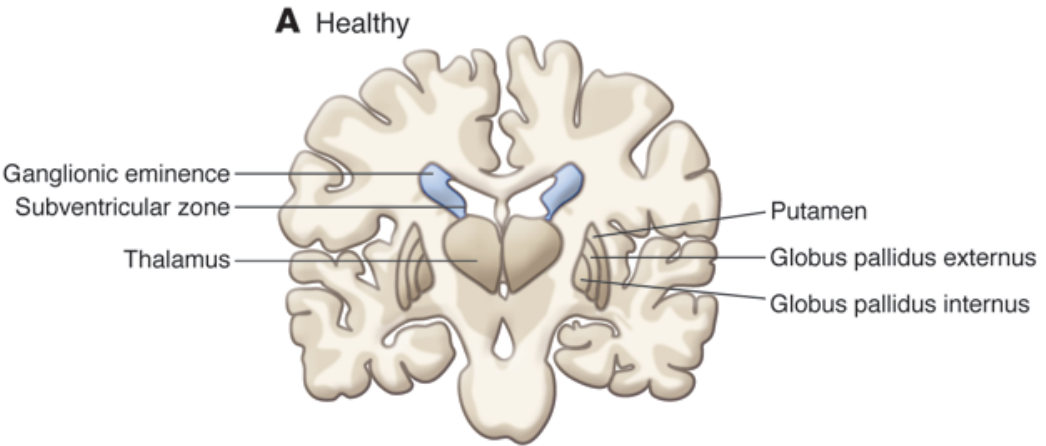

B Noncystic PVL

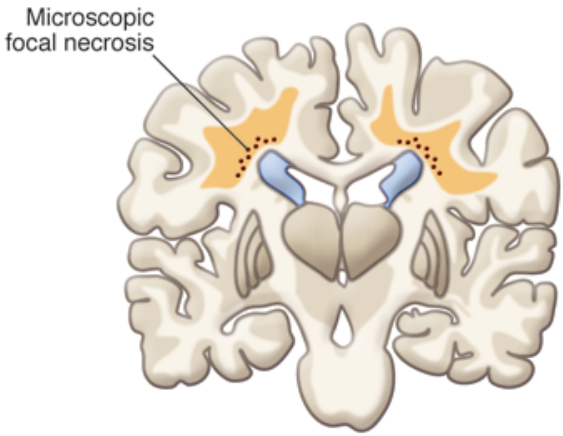

D IVH

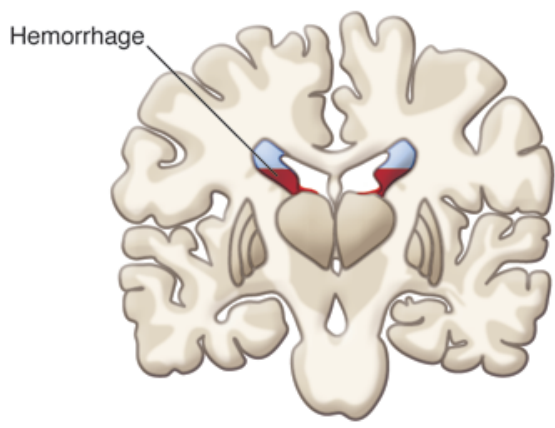

cifically, the authors developed a bitransgenic mouse in which selective induction and deinduction of a secreted VEGF decoy receptor is accomplished by the presence or absence of tetracycline in the drinking water. In this model, expression of the decoy VEGF receptor is driven by the neuronal tissue-specific calmodulin kinase II $\alpha$ promoter, allowing selective blockade of VEGF signaling within the brain at discrete developmental stages and for specified intervals. While transient VEGF blockade in early development (E9.5) resulted in nonviable pups with severe cortical abnormalities, blockade at later time points resulted in less severe phenotypes. By E13.5-E14.5, VEGF blockade resulted in a spatially restricted apoptosis that involved only a small subset of cells within the ganglionic eminence, an area adjacent to the lateral ventricle that
Figure 1. Types of brain injuries frequently encountered in premature neonates. Premature infants are prone to a variety of brain injuries. (A) A cross section of a healthy brain shows areas that are associated with injuries of prematurity. (B) The most common injury is noncystic PVL, which presents as a diffuse cerebral white-matter injury (orange) accompanied by microscopic focal necrotic lesions. (C) Cystic PVL is a more severe disease that, in addition to diffuse white-matter injury, presents with macroscopic necrotic lesions. (D) IVH, which occurs with much less frequency than PVL in premature infants, is characterized by bleeding (red) into the ventricular system of the brain. (E) The most extreme cases of IVH are associated with periventricular hemorrhagic infarction into the white matter.

poral window of vulnerability. Licht, DorWollman, et al. note a lack of vessels in the affected regions of their model that corresponds to the striking paucity of arteries in histologic sections of infants with PVL (14). This model is also consistent with data from histologic sections of human fetal and postnatal brain specimens that show region-specific waves of VEGF immunoreactivity (15).

Licht, Dor-Wollman, and colleagues speculate that the biology of PVL parallels that of retinopathy of prematurity (ROP), wherein hyperoxia exposure suppresses VEGF (via repression of HIF), which induces apoptosis of nascent vascular endothelial cells (16). Interestingly, a recent publication by Yuen et al. shows that in postnatal murine development, preOLs play a role in vascularization of white matter. Hypoxic HIF stabilization in these precursors directly upregulates transcription of the WNT ligands 7a and $7 \mathrm{~b}$, which then act in an autocrine manner to delay oligodendrocyte differentiation and myelination as well as in a paracrine fashion to stimulate angiogenesis in the corpus callosum (17). This postnatal whitematter vasculogenesis is predominantly at the capillary level (18) and distinct from the early angiogenesis that characterizes the VEGF-dependent window proposed by Licht, Dor-Wollman, et al. However, postnatal vasculogenesis in white matter does substantiate an ongoing role for HIF-mediated oxygen sensitivity in the coordination and direction of cerebral vascular development in particular as well as developmental vascularization in general (19).

\section{Recommendations}

It is now clear from the collective publication of the Neonatal Oxygenation Prospective 
Meta-analysis Collaboration (NEOPROM) studies of high versus low oxygen saturation targets that management aimed to minimize hyperoxic exposure in premature infants can reduce the incidence of ROP (20-22). It is less clear what effect lower target saturations may have on neurodevelopmental outcomes, however. While neither the Canadian Oxygen Trial (COT) nor the Study to Understand Prognoses and Preferences for Outcomes and Risks of Treatments (SUPPORT) trial demonstrated a difference in the primary outcome of death or severe neurodevelopmental disability at 18 to 24 months, metaanalysis of the five trials found increased mortality in the low-saturation group (22). The low-saturation groups also had an increased incidence of necrotizing enterocolitis (NEC), a trend in the metaanalysis that was not significant in any of the individual trials. There was no substantial difference in other neurologic end points evaluated; however, these specific outcome measures were not uniform and generally encapsulated only the most blatant injuries, such as high-grade IVH. The findings by Licht, Dor-Wollman, et al. highlight the need for attention not just to IVH but also to a wider range of brain injuries in premature infants that potentially stem from exposure to hyperoxic conditions.

Perhaps most importantly, the insights from the paper by Licht, Dor-Wollman, and colleagues emphasize a potentially limited time frame of highest developmental risk. This model suggests that there is a defined transition point to VEGF independence and, by inference, decreased hyperoxia vulnerability, with respect to periventricular vascularity. Infants enrolled in the NEOPROM studies randomized to high- or low-saturation targets were maintained at their oxygen targets through 36-weeks gestational age, but this may far outlive the therapeutic benefit of relative hypoxia. At 29- to 32-weeks gestational age, when the incidence of NEC is reaching its peak (23), there may be no further neurovascular benefit from this strategy. Thus, it may be more appropriate to consider a tiered approach to oxygen saturation guidelines in premature infants that vary with their gestational age. Infants between 24- and 28-weeks gestational age, for example, could be targeted to lower $\mathrm{O}_{2}$ saturations, with gradual increases to normal pediatric values after 28-weeks gestational age. Such an approach would therefore enable premature infants to reside in a physiologically hypoxic environment during the highly oxygen-sensitive developmental windows regulating cerebral and retinal vascularization, while preventing their exposure to potentially pathological hypoxia during later gestational ages at which diseases such as NEC are more likely to occur. The publication by Licht, Dor-Wollman, et al. therefore underscores the importance of developing more nuanced approaches to the use of oxygen therapy in the intensive care nursery than currently practiced.

Proper development requires precisely coordinated interactions between the embryo/fetus and its environment. A better understanding of the fundamental environmental mechanisms that drive mammalian development in utero will therefore help neonatologists devise more physiologically relevant treatment modalities to improve the care of premature neonates ex utero. Importantly, there is a growing urgency for increased basic research in this area as technological improvements in our abilities to sustain the lives of increasingly premature and small-for-gestationalage infants outstrip our abilities to ensure a meaningful quality of life for this incredibly at-risk population.

Address correspondence to: Emin Maltepe, Associate Professor of Pediatrics and Biomedical Sciences, The Center for Reproductive Sciences, Division of Neonatology, 513 Parnassus Ave. HSE-1422, Box 1346, San Francisco, California 941431346, USA. Phone: 415.476.9313; E-mail: emin.maltepe@ucsf.edu.

1. Berry JG, et al. Inpatient growth and resource use in 28 children's hospitals: a longitudinal, multi-institutional study. JAMA Pediatr. 2013;167(2):170-177.

2. Back SA, Riddle A, McClure MM. Maturationdependent vulnerability of perinatal white matter in premature birth. Stroke. 2007; 38(2 suppl):724-730.

3. Moore T, et al. Neurological and developmental outcome in extremely preterm children born in England in 1995 and 2006: the EPICure studies. BMJ. 2012;345:e7961.

4. Volpe JJ. Cerebral white matter injury of the premature infant - more common than you think. Pediatrics. 2003;112(1 pt 1):176-180.

5 . Volpe JJ. Brain injury in premature infants: a complex amalgam of destructive and developmental disturbances. Lancet Neurol. 2009;8(1):110-124.

6. Inder TE, Anderson NJ, Spencer C, Wells S,
Volpe JJ. White matter injury in the premature infant: a comparison between serial cranial sonographic and MR findings at term. AJNR Am J Neuroradiol. 2003;24(5):805-809.

7. Woodward LJ, Anderson PJ, Austin NC, Howard $\mathrm{K}$, Inder TE. Neonatal MRI to predict neurodevelopmental outcomes in preterm infants. N Engl JMed. 2006;355(7):685-694.

8. Khwaja O, Volpe JJ. Pathogenesis of cerebral white matter injury of prematurity. Arch Dis Child Fetal Neonatal Ed. 2008;93(2):F153-F161.

9. Rorke LB. Anatomical features of the developing brain implicated in pathogenesis of hypoxicischemic injury. Brain Pathol. 1992;2(3):211-221.

10. Inage YW, Itoh M, Takashima S. Correlation between cerebrovascular maturity and periventricular leukomalacia. Pediatr Neurol. 2000;22(3):204-208.

11. Takashima S, Armstrong DL, Becker LE. Subcortical leukomalacia. Relationship to development of the cerebral sulcus and its vascular supply. Arch Neurol. 1978;35(7):470-472.

12. Elitt CM, Rosenberg PA. The challenge of understanding cerebral white matter injury in the premature infant. Neuroscience. 2014;276:216-238.

13. Licht T, Dor-Wollman T, Ben-Zvi A, Rothe G, Keshet E. Vessel maturation schedule determines vulnerability to neuronal injuries of prematurity. J Clin Invest. 2015;125(3):1319-1328.

14. Takashima S, Tanaka K. Development of cerebrovascular architecture and its relationship to periventricular leukomalacia. Arch Neurol. 1978;35(1):11-16.

15. Arai Y, Deguchi K, Takashima S. Vascular endothelial growth factor in brains with periventricular leukomalacia. Pediatr Neurol. 1998;19(1):45-49.

16. Alon T, Hemo I, Itin A, Pe'er J, Stone J, Keshet E. Vascular endothelial growth factor acts as a survival factor for newly formed retinal vessels and has implications for retinopathy of prematurity. Nat Med. 1995;1(10):1024-1028.

17. Yuen TJ, et al. Oligodendrocyte-encoded HIF function couples postnatal myelination and white matter angiogenesis. Cell. 2014;158(2):383-396.

18. Harb R, Whiteus C, Freitas C, Grutzendler J. In vivo imaging of cerebral microvascular plasticity from birth to death. JCereb Blood Flow Metab. 2013;33(1):146-156.

19. Dunwoodie SL. The role of hypoxia in development of the Mammalian embryo. Dev Cell. 2009;17(6):755-773.

20. BOOST II United Kingdom Collaborative Group, et al. Oxygen saturation and outcomes in preterm infants. $N$ Engl J Med. 2013;368(22):2094-2104.

21. SUPPORT Study Group of the Eunice Kennedy Shriver NICHD Neonatal Research Network, et al. Target ranges of oxygen saturation in extremely preterm infants. $\mathrm{N} \mathrm{Engl} \mathrm{JMed}$. 2010;362(21):1959-1969.

22. Saugstad OD, Aune D. Optimal oxygenation of extremely low birth weight infants: a meta-analysis and systematic review of the oxygen saturation target studies. Neonatology. 2014;105(1):55-63.

23. Sharma R, Hudak ML. A clinical perspective of necrotizing enterocolitis: past, present, and future. Clin Perinatol. 2013;40(1):27-51. 